\title{
Phase behaviour of colloids with short-range repulsions plus nonadsorbing polymer chains
}

\author{
Kitty van Gruijthuijsen, $\dagger^{\mathrm{a}}$ Remco Tuinier, ${ }^{\text {*bc }}$ Joseph M. Brader ${ }^{\mathrm{d}}$ and Anna Stradner ${ }^{\mathrm{e}}$
}

\begin{abstract}
The colloidal gas-liquid phase behaviour has been studied for aqueous mixtures of well-defined spherical particles with shortranged repulsions mixed with relatively monodisperse poly(ethylene oxide) polymers. We show that our set of experimental phase diagrams are in quantitative agreement with theoretical predictions using generalized free volume theory [G. J. Fleer and R. Tuinier, Phys. Rev. E, 2007, 76, 041802]. The determination of the equilibrium composition of coexisting phases reveals qualitative deviations between the two, for which we propose a tentative explanation.
\end{abstract}

\section{Introduction}

The properties of many food products, paints, biological systems like living cells and other complex materials are related to the intrinsic immiscibility of colloids and non-adsorbing polymers at high concentrations. ${ }^{1}$ This incompatibility originates from depletion interactions. The excluded volume between the colloids and nonadsorbing polymer chains results in effective depletion zones around the colloids. Upon overlap of these depletion layers the polymeric osmotic pressure becomes unbalanced and leads to an effective attraction between the colloids. ${ }^{2}$ A quantitative understanding of this phenomenon is paramount to control the processing of such mixtures. Moreover, it offers interesting routes to design products with novel properties. ${ }^{3}$

${ }^{a}$ Adolphe Merkle Institute, University of Fribourg, Route de l'ancienne Papeterie 1, Marly, Switzerland. Fax: +4126300 9642; Tel: +41792431753

${ }^{b}$ Van 't Hoff Laboratory for Physical and Colloid Chemistry, Debye Institute, Utrecht University, Utrecht, The Netherlands. E-mail: R.Tuinier@uu.nl; Fax: +31 30253 3870; Tel: +31302533406

'DSM ChemTech Center, Advanced Chemical Engineering Solutions (ACES), P.O. Box 18, $6160 \mathrm{MD}$ Geleen, The Netherlands

${ }^{d}$ Soft Matter Theory, University of Fribourg, Chemin du Musée 3, Fribourg, Switzerland. E-mail: Joseph.Brader@unifr.ch; Fax: +41 26300 9758; Tel: +41 26 3008914

${ }^{e}$ Physical Chemistry, Lund University, Getingevägen 60, Lund, Sweden. E-mail: Anna. Stradner@fkem1.lu.se; Fax: +46 46222 4413; Tel: +46 462228214

$\dagger$ Current address: Firmenich SA, Geneva, Switzerland. E-mail: Kitty.vanGruijthuijsen@gmail.com
It is well understood that the range of the attractive potential is determined by the size ratio between the polymers with radius of gyration $R_{\mathrm{g}}$ and the colloids with radius $R$, defined as $\gamma=R_{\mathrm{g}} / R$, while the depth is set by the osmotic pressure of the polymer solution. The equilibrium phase diagram of such mixtures with variable $\gamma$ is well described by Generalized Free Volume Theory (GFVT), ${ }^{4,5}$ which is based upon a semi-grand canonical description of colloids dispersed in a polymer solution. ${ }^{6}$ The colloids interact as hard spheres with radius $R$; the polymers follow scaling laws for coils with mean field or excluded volume interactions; and colloids and polymers mutually interact as hard spheres with radii $R$ and the depletion thickness $\delta$, respectively. The latter converges to a value close to $R_{\mathrm{g}}$ for dilute polymer solutions, and to the correlation length in the polymer solution, $\xi$, above the polymer overlap concentration, $c^{*}{ }^{4}$

GFVT can be extended to include the effect of screened Coulomb repulsions between the colloids, integrated into an effective colloid-colloid interaction radius, $R_{\text {eff. }}{ }^{7-9}$ Several experimental studies of colloid-polymer mixtures confirm the qualitative effects of additional repulsions, ${ }^{9-15}$ as well as the quantitative shift of the binodal towards higher polymer concentrations. ${ }^{9}$ We now present a set of experimental phase diagrams of aqueous dispersions containing charged polystyrene (PS) colloids and neutral poly(ethylene oxide) (PEO) polymer. The determination of the binodal over a larger range of colloid volume fractions reveals an additional qualitative deviation between the experiment and GFVT.

\section{Materials and methods}

We synthesized polystyrene colloids that are stabilized against van der Waals attractions by a grafted shell of Tween 80 as described previously. ${ }^{16}$ Poly(ethylene oxide) (PEO) polymer was purchased from Polymer Source and used without further purification. $\mathrm{NaCl}$ was used throughout to set the added salt concentration, $c_{\mathrm{s}}$. The characteristics of the colloids and the polymers are listed in Table 1 . The effective interactions 
Table 1 Characteristics of the synthesized colloids (stock solution with $c_{s}=20$ $\mathrm{mM}$ ) and the purchased polymers.

\begin{tabular}{llllll}
\hline Polystyrene colloids & \multicolumn{2}{l}{ Poly(ethylene oxide) polymer } \\
\hline$c^{a}\left[\mathrm{~g} \mathrm{~L}^{-1}\right]$ & 492 & $M_{\mathrm{n}}{ }^{d}\left[\mathrm{~kg} \mathrm{~mol}^{-1}\right]$ & 430 & 203 & 83 \\
$R_{\mathrm{h}}{ }^{b}[\mathrm{~nm}]$ & $65 \pm 11$ & $M_{\mathrm{W}}{ }^{d}\left[\mathrm{~kg} \mathrm{~mol}^{-1}\right]$ & 480 & 231 & 90 \\
$R^{c}[\mathrm{~nm}]$ & 59 & $R_{\mathrm{g}}{ }^{2}=0.99 M_{\mathrm{w}}{ }^{0.60}[\mathrm{~nm}]$ & 40 & 26 & 15 \\
$Z_{\mathrm{eff}}{ }^{c}$ & 530 & $c^{*}=3 M_{\mathrm{w}} / 4 \pi N_{\mathrm{A}} R_{\mathrm{g}}{ }^{3}\left[\mathrm{~g} \mathrm{~L}^{-1}\right]$ & 2.9 & 5.2 & 15 \\
$\phi^{c}$ & 0.51 & $R_{\mathrm{g}} / R$ & 0.68 & 0.44 & 0.25
\end{tabular}

${ }^{a}$ Concentration, measured with a Moisture Analyzer MA35 (Sartorius). ${ }^{b}$ Hydrodynamic radius, measured with dynamic light scattering on a 3D LS Spectrometer (LS Instruments) at an angle of $90^{\circ}$ at $c=0.05 \mathrm{~g}$ $\mathrm{L}^{-1} \cdot{ }^{17,18}{ }^{c}$ Hard sphere radius, $R$, effective surface charges, $Z_{\text {eff }}$ (uncertainty $\sim 10 \%$ ), and colloid volume fraction, $\phi$, based on SAXS measurements. ${ }^{d}$ Number and weight average molar mass, according to the supplier. ${ }^{e}$ Relation between $R_{\mathrm{g}}(\mathrm{nm})$ and $M_{\mathrm{w}}\left(\mathrm{kg} \mathrm{mol}^{-1}\right)$ deduced from a series of literature data, spanning four orders of magnitude in $M_{\mathrm{w}} \cdot{ }^{19-24}$

between the colloids were characterized via measurements of the structure factor using small angle X-ray scattering (SAXS) at the cSAXS beamline at the Paul Scherrer Insitute, Villigen, Switzerland, following ref. 16. We used $11.2 \mathrm{eV}$ X-rays, corresponding to a wavelength of $\lambda=0.111 \mathrm{~nm}$, with which we could access a $q$-range of $0.009-1 \mathrm{~nm}^{-1}$. The measurements were performed in $1 \mathrm{~mm}$ glass mark-tubes (Hilgenberg). The 2D scattering patterns were azimuthally averaged to obtain the scattering intensities, $I(\boldsymbol{q})$, as a function of the scattering vector $\boldsymbol{q}=4 \pi \sin (\theta / 2) / \lambda$, with $\theta$ the scattering angle (Fig. 2).

To construct our colloid-polymer phase diagrams, we mixed appropriate amounts of stock solutions of the colloids and polymers, both in $20 \mathrm{mM}$ salt, and additional quantities of demineralised water and more concentrated salt solutions.
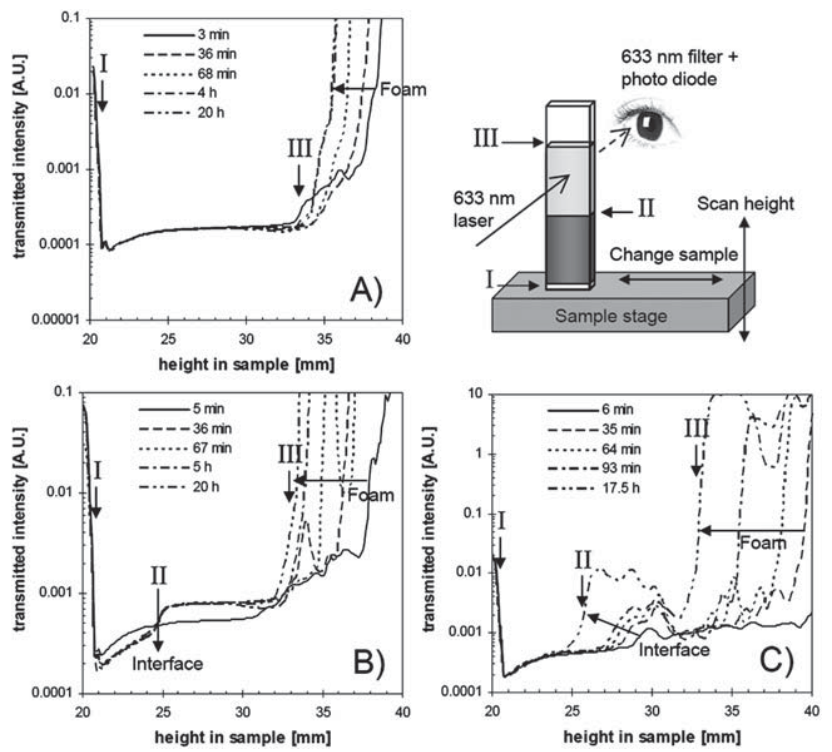

Fig. 1 Transmission height profiles for samples in a dilution series $\left(c_{s}=30 \mathrm{mM}\right)$ at different times after mixing, as listed in the legends. Samples correspond to (A) a homogeneous mixture, (B) fluid-fluid phase separation, and (C) a gel. The schematic depicts the principle of the Profiler. Roman numbers relate real sample positions (schematic) to measured heights (graphs).



Fig. 2 SAXS scattering intensities of the colloids in $\mathrm{H}_{2} \mathrm{O}$ at $20{ }^{\circ} \mathrm{C}$. The legend indicates colloid concentrations, full symbols are in $50 \mathrm{mM}$, open symbols in 20 $\mathrm{mM}$, plus signs in $3 \mathrm{mM}$, minus signs in $5 \mathrm{mM}$, and crosses in $8 \mathrm{mM} \mathrm{NaCl}$ solutions. The solid line is the SAXS form factor as discussed in the text. For clarity, the data for $c=2 \times 10^{2}, 3 \times 10^{2}$ and $492 \mathrm{~g} \mathrm{~L}^{-1}$ are shifted upwards by a factor of $2^{2}, 2^{4}$ and $2^{7}$, respectively. The image shows part of the $2 \mathrm{D}$ scattering pattern of the sample with $c=492 \mathrm{~g} \mathrm{~L}^{-1}$ in $20 \mathrm{mM}$ salt (open squares in the graph), where the arrows point out some of the typical reflections due to crystallization.

Concentrations are expressed in $\mathrm{g} \mathrm{L}^{-1}$ for both the colloids, $c$, and polymers, $c_{\mathrm{p}}$. The mixtures were directly weighed into glass rectangular cells with dimensions $5 \times 10 \mathrm{~mm}^{2}$ that could be closed with a Teflon stopper (Yixing Zhicheng Material). After a $30 \mathrm{~s}$ homogenization step on a vortex mixer (MS1 Minishaker, IKA), the samples were followed in time with the Profiler (in-house design, schematic Fig. 1). It measures the transmission of laser light with a wavelength $\lambda=633 \mathrm{~nm}$ as a function of height in the sample cell, which we refer to as the transmission height profile (THP). We designed a sample stage mounted on two high-precision motors: a vertical motor to scan through the sample and a horizontal motor to move between five sample positions. Since we measured four to five samples in parallel, this gave us timeresolved information on a $30 \mathrm{~min}$ time scale.

Samples were considered homogeneous fluids in the absence of an interface; being phase separated into two fluids when an interface appeared at a certain height in the sample, which became clearer with time without changing its position, and a gel when an interface appeared at the top of the sample and moved down with time. Fig. 1A-C show some typical profiles. Samples that had phase separated after $24 \mathrm{~h}$ were measured with the Profiler once more, before being diluted with a small aliquot of $\mathrm{NaCl}$ solution at the same concentration as the salt concentration of the solvent. In all samples that were used to determine the binodal, either directly or using the lever rule, the foam layer had fully disappeared at the moment of measurement at $t=24 \mathrm{~h}$. Due to the high turbidity of both phases close to the binodal the samples had to be assessed visually with LED illumination to determine whether or not phase separation had taken place.

\section{Results and discussion}

\section{Colloid characterization}

To compare our experimental phase diagrams to GFVT calculations, we need to convert experimentally accessible quantities 

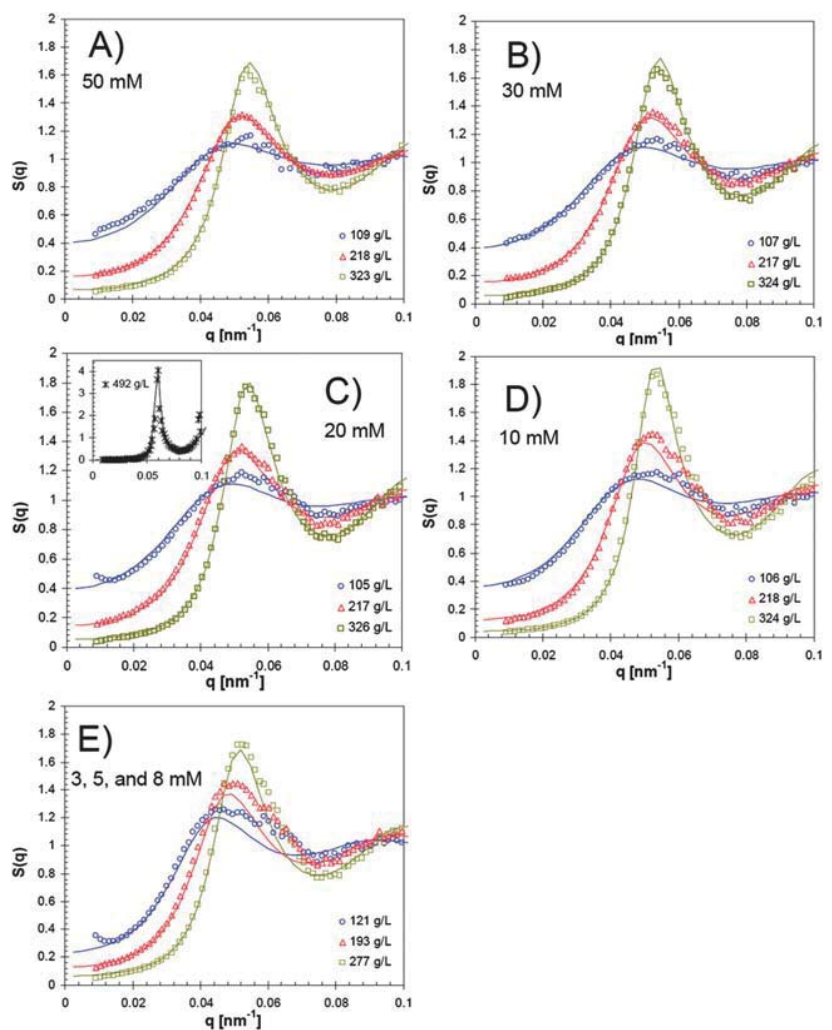

Fig. 3 Structure factors of the colloids at $20^{\circ} \mathrm{C}$, as measured by SAXS. Samples with $c=10^{2} \mathrm{~g} \mathrm{~L}^{-1}, 2 \times 10^{2} \mathrm{~g} \mathrm{~L}^{-1}, 3 \times 10^{2} \mathrm{~g} \mathrm{~L}^{-1}$, and $492 \mathrm{~g} \mathrm{~L}^{-1}$ are depicted as blue, red, green, and black symbols respectively. Salt concentrations in the solvent are (A) $50 \mathrm{mM}$, (B) $30 \mathrm{mM}$, (C) $20 \mathrm{mM}$, (D) $10 \mathrm{mM}$, and (E) $3 \mathrm{mM}$ for $\mathrm{c}=121 \mathrm{~g} \mathrm{~L}^{-1}$, $5 \mathrm{mM}$ for $\mathrm{c}=193 \mathrm{~g} \mathrm{~L}^{-1}$, and $8 \mathrm{mM}$ for $\mathrm{c}=277 \mathrm{~g} \mathrm{~L}^{-1}$. Solid lines are the theoretical calculations using the Percus-Yevick closure of the Ornstein-Zernike equation for colloids interacting via electrostatic repulsions.

like the colloid concentration, $c$, expressed in $\mathrm{g} \mathrm{L}^{-1}$ to reduced quantities like the colloid volume fraction, $\phi$, typically used in theoretical descriptions. To this end, we measure scattering intensities of a colloid/salt concentration series with SAXS, as shown in Fig. 2. The suppressed forward scattering and development of a structure factor peak around $q=0.05-0.06 \mathrm{~nm}^{-1}$ indicate an increased colloid structuring with increasing colloid concentrations and/or decreasing salt concentrations. The clear form factor oscillations at higher $q$-values point to a rather low colloid polydispersity. The sharp peaks occurring in the stock solution, with $c=492 \mathrm{~g} \mathrm{~L}^{-1}$, suggest that it was partially crystallized at the time of measurement. In fact, in the 2D scattering pattern of this sample the ring pattern is no longer fully homogeneous, but features localized spots, which are typically related to crystallites in the sample. As an illustration, some of these spots are indicated with arrows in Fig. 2. The high colloid concentration of $c=492 \mathrm{~g} \mathrm{~L}^{-1}$ is indeed close to the freezing line $\phi_{\mathrm{f}}=0.494$ for monodisperse hard spheres. ${ }^{25}$ It is therefore not surprising that the system crystallizes at a salt concentration of $c_{\mathrm{s}}=20 \mathrm{mM}$, where the additional Coulomb repulsion is not completely screened and results in a larger effective hard sphere volume fraction.

We extract structure factors, $S(q)$, from the scattered intensities by normalization with the concentration and division with the form factor, $P(q)$. The measurement of a dilute sample with $c=18 \mathrm{~g} \mathrm{~L}^{-1}$, where $S(q) \approx 1$, is too close to the background scattering to properly resolve the form factor minima and maxima. To minimize effects from the strong background scattering, we calculate a theoretical structure factor for the sample with $c=218 \mathrm{~g} \mathrm{~L}^{-1}$ and $c_{\mathrm{s}}=50 \mathrm{mM}$. In our previous work, we used the Modified Penetrating Background Mean Random Sphere Approximation together with a decoupling approximation to calculate the structure factor of slightly polydisperse spheres. ${ }^{16}$ For a polydispersity below $10 \%$, its effects are mostly visible as an incoherent contribution to the forward scattering, which we neglect here. Instead, we use the Percus-Yevick closure of the Ornstein-Zernike equation of colloids interacting via electrostatic repulsions. We verified that for the studied electrostatic screening conditions, very similar results were obtained with both approaches. To fit the dataset as a whole, we iteratively: (1) divide the experimental data of the sample with $c=218 \mathrm{~g} \mathrm{~L}^{-1}$ and $c_{\mathrm{s}}=50 \mathrm{mM}$ by a theoretical structure factor to obtain the SAXS form factor; (2) use the latter to calculate experimental and theoretical structure factors for the whole series; and (3) adjust the initial modelling parameters if necessary to describe the dataset as a whole.

The modelling parameters, being the colloid radius $R$, the ratio $c / \phi$ and the effective surface charge $Z_{\text {eff }}$, that describe the dataset well are $R=59 \mathrm{~nm}, c / \phi=957 \mathrm{~g} \mathrm{~L}^{-1}$, and $Z_{\text {eff }}=530$. The experimental data together with the calculated $S(q)$ s are shown in Fig. 3. Note that a variation in $Z_{\text {eff }}$ of $\pm 10 \%$ does not markedly affect the quality of the modelled $S(q)$ s.

\section{Phase diagram with effective hard spheres}

To establish the phase diagram of colloid-polymer mixtures with $\gamma=0.68$, we measure five dilution series of mixtures of the colloids with PEO polymer with $R_{\mathrm{g}}=40 \mathrm{~nm}$. The resulting experimental phase diagrams are summarized in Fig. 4 for salt concentrations of $20 \mathrm{mM}, 30 \mathrm{mM}$, and $50 \mathrm{mM}$ (corresponding to a narrow range of Debye lengths from $\sim 2.1$ to $1.3 \mathrm{~nm}$ ). We first focus on the mixtures that are closest to being hard spheres, the ones with $c_{\mathrm{s}}=50 \mathrm{mM}$ depicted as black squares and crosses in Fig. 4. As expected, we find homogeneous samples at low colloid and polymer concentrations and fluid-fluid phase separation at higher concentrations, which is eventually arrested to form gels. ${ }^{26}$ The colloids are sufficiently monodisperse to form crystals, as demonstrated in Fig. $2 .{ }^{27}$ We indeed observe three-phase coexistence in between the gel and the fluid-fluid region. ${ }^{28}$ However, we have not consistently verified possible crystallization for all samples (i.e. wait several days for crystallization to occur), nor have we tested whether these phases truly were a gas, a liquid, and a solid.

To compare the results with GFVT calculations, the abscissae are converted to volume fractions using our SAXS results, i.e. $\phi$ $=c / 957 \mathrm{~g} \mathrm{~L}^{-1}$, and the ordinate is normalized by the PEO overlap concentration $c^{*}=2.9 \mathrm{~g} \mathrm{~L}^{-1}$. The THPs are used to extract the height ratio between the upper and lower phase. We then apply the lever rule for two component mixtures to calculate the composition of the coexisting phases. ${ }^{29}$ Since we measured rather extended dilution series with multiple dilution 


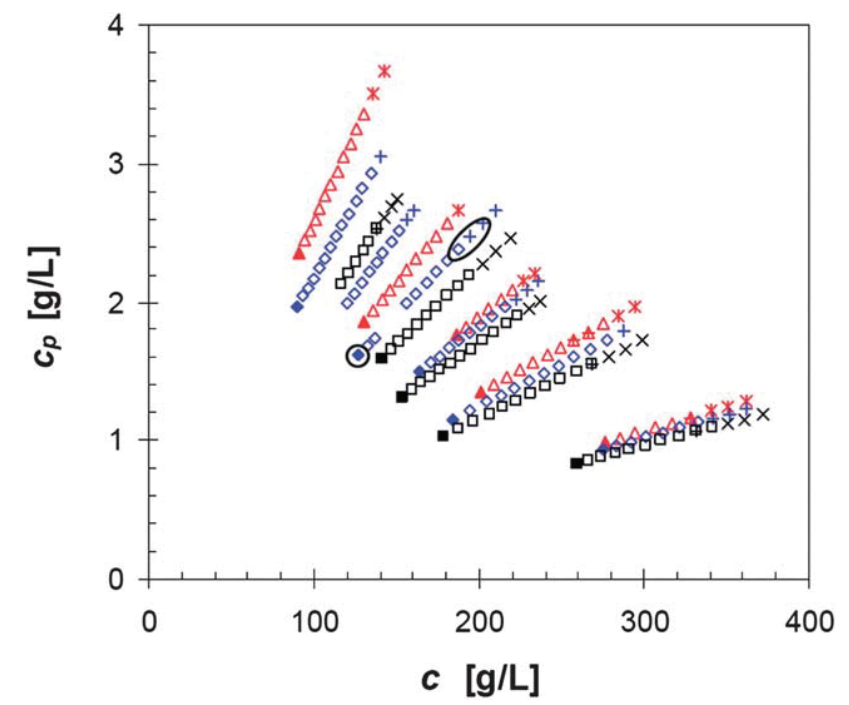

Fig. 4 Experimental phase diagram of colloid-polymer mixtures with $\gamma=0.68$ and $c_{\mathrm{s}}=50 \mathrm{mM}$ (black squares and crosses), $30 \mathrm{mM}$ (blue diamonds and plus symbols), and $20 \mathrm{mM}$ (red triangles and asterisks). Asterisk, cross and plus symbols correspond to gels, open symbols to fluid-fluid phase separation, and filled symbols to homogeneous mixtures. The THPs of the encircled samples are shown in Fig. 1.

steps, we expect that there will be significant errors in the computed sample compositions. The uncertainty in the final data is estimated to be \pm 0.05 in $\phi$ and \pm 0.1 in $c_{\mathrm{p}} / c^{*}$. The experimental results are shown as symbols in Fig. 5 and overlaid with GFVT calculations of the gas-liquid coexistence curve for mixtures of hard spheres with excluded-volume polymers with a size ratio of $\gamma=0.68 .^{5,28}$ We find excellent quantitative agreement. Phase separation is often considered a negative feature in



Fig. 5 Phase diagram with normalized axes of colloid-polymer mixtures with $\gamma=$ 0.68 and $c_{\mathrm{s}}=50 \mathrm{mM}$. Filled squares depict experimental binodal points, open squares coexisting fluid phases determined with the lever rule, and filled circles midpoints of experimental tie lines. The solid line is the GFVT result for $\gamma=0.68$ and $m=$ 1 with the open circle being the critical point. The dashed line is a theoretical tie line, and the dotted line an experimental one for a hypothetical starting mixture denoted with $\mathrm{X}$; error bars indicate the uncertainty in the composition of the corresponding experimental coexisting phases (grey filled squares). applications of colloid-polymer mixtures. To prevent product instability, a good prediction of the binodal can thus facilitate product formulation.

Interestingly, the thermodynamically unstable region above the binodal can also be utilized to form gels. ${ }^{26,30-32}$ To rationalize gelation experiments, it is useful to know the tie lines of the equilibrium phase diagram, i.e. the composition of coexisting phases and their relative volumes. ${ }^{33}$ If we compare our experimental tie lines to the theoretical ones, being respectively the dotted and dashed line in Fig. 5, we find them in reasonable agreement. The polymer concentration in the coexisting colloidpoor phase is overestimated by the theory, resulting in a slightly steeper theoretical tie line, but falls within the estimated uncertainty of the experimental coexistence points. Moreover, if we extrapolate the mid-points of the experimental tie lines, depicted as filled circles in Fig. 5, we end up close to the theoretical critical point. Along this line, the two phases will occupy equal volumes and will both be space spanning during the initial stages of the phase separation process.

\section{Phase diagrams with repulsive colloids}

As expected, when the repulsions are increased by a reduction of the salt concentration, more polymer is needed to induce phase separation, shown by the blue diamonds and red triangles in Fig. 4. Notably at low colloid concentrations the onset of phase separation as well as the gel boundary shifts to significantly higher polymer concentrations with decreasing salt concentration.

Also for $c_{\mathrm{s}}=20 \mathrm{mM}$ and $30 \mathrm{mM}$ we use the THPs to extract the composition of coexisting phases using the dilution series and the lever rule. ${ }^{29}$ The results are shown in Fig. 6 as open

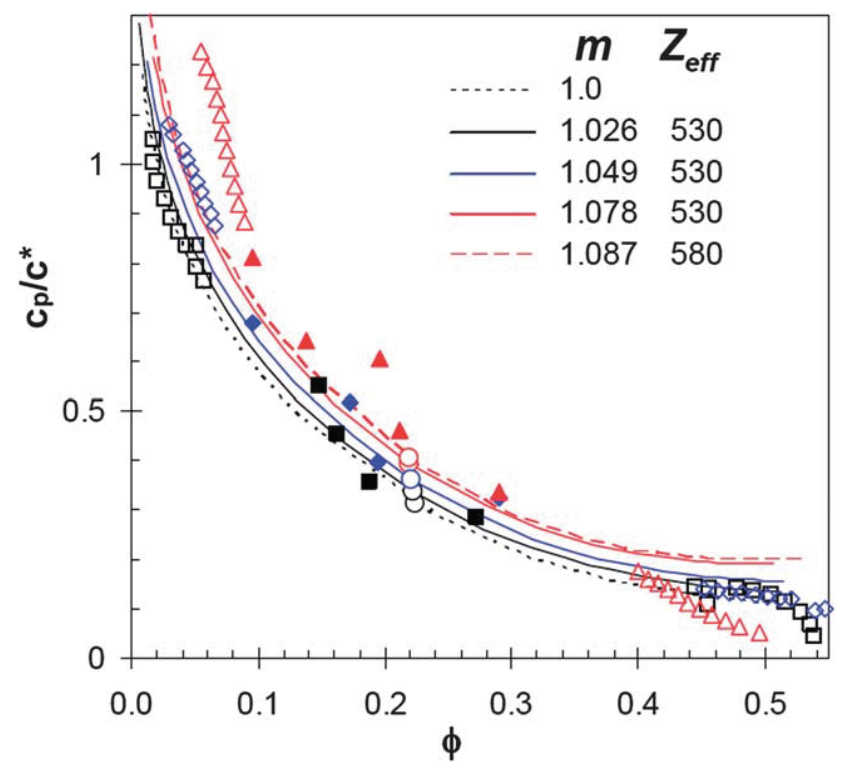

Fig. 6 Phase diagram with normalized axes of colloid-polymer mixtures with $\gamma$ $=0.68$ and $c_{\mathrm{s}}=50 \mathrm{mM}$ (black squares and lines), $30 \mathrm{mM}$ (blue diamonds and lines), and $20 \mathrm{mM}$ (red triangles and lines). Filled symbols depict experimental binodal points, open symbols coexisting phases determined with the lever rule, and circles theoretical critical points. The lines are GFVT results for $\gamma=0.68$ and values for $m$ and $Z_{\text {eff }}$ are as listed in the legend. 
symbols. Strikingly, reduced screening of the charges seems to shift the coexistence points at low colloid volume fractions to considerably higher polymer concentrations, with a less clear effect on the high volume fraction tail of the coexistence curve. We apply the extension of GFVT to charged colloids, ${ }^{7,8}$ where screened Coulomb repulsions are described by a Yukawa type potential, the strength of which is set by the number of effective surface charges, $Z_{\text {eff }}$, and the range is determined by the salt concentration in the bulk, $c_{\mathrm{s}}:{ }^{34}$

$$
U_{\mathrm{ES}}(r)=\frac{l_{\mathrm{B}} Z_{\mathrm{eff}}^{2} \exp (-\kappa(r-2 R))}{(1+\kappa R)} \quad \text { for } r>2 R
$$

where $l_{\mathrm{B}} \approx 0.7 \mathrm{~nm}$ is the Bjerrum length in water at a temperature of $T=293{ }^{\circ} \mathrm{C}$, and $r$ the center-to-center colloid distance. The inverse Debye screening length, $\kappa$, is given by:

$$
\kappa=\sqrt{\frac{3 l_{B} \phi\left|Z_{\text {eff }}\right|}{R^{3}(1-\phi)}+8 \pi l_{\mathrm{B}} c_{\mathrm{s}} N_{\mathrm{A}}}
$$

with $c_{\mathrm{s}}$ the salt concentration in the continuous aqueous phase in $\mathrm{mM}\left(\mathrm{mol} \mathrm{m}^{-3}\right)$, and $N_{\mathrm{A}}$ Avogadro's number. The extended GFVT accounts for additional repulsions by integration over the repulsive potential into an effective colloid-colloid interaction radius, $R_{\text {eff }}$, following: ${ }^{8}$

$$
\frac{R_{\mathrm{eff}}}{R}=m^{1 / 3}=1+0.5 \int_{2 R}^{\infty}\left[1-\exp \left[-U_{\mathrm{ES}}(r)\right]\right] \mathrm{d} r
$$

In the thermodynamic framework of GFVT all colloid volume fractions relevant to colloid-colloid interactions are replaced by an effective colloid volume fraction $\phi_{\text {eff }}=m \phi$. For dilute colloids and $Z_{\text {eff }}=530$, we calculate $m=1.026$ for $c_{\mathrm{s}}=50 \mathrm{mM}, m=1.049$ for $c_{\mathrm{s}}=30 \mathrm{mM}$, and $m=1.078$ for $c_{\mathrm{s}}=20 \mathrm{mM}$. We use these values to compute the binodal for colloids which mutually interact as hard spheres with radius $R_{\text {eff }}=m^{1 / 3} R$, but which interact with the excluded-volume polymers with radius $R .^{7,8} \mathrm{We}$ find that with increasing $m$-values the GFVT binodals shift upwards over the whole range of colloid volume fractions, as shown in Fig. 6. Although the predictions seem to quantitatively capture the shift to higher polymer concentrations, they do not qualitatively reflect the experimental trends that we observed over the whole range of volume fractions.

As mentioned above, our approach to determine $Z_{\text {eff }}$ values from $S(q)$ measurements results in an uncertainty of about $10 \%$ in $Z_{\text {eff. }}$ The effect of a $10 \%$ increase of $Z_{\text {eff }}$ for $c_{\mathrm{s}}=20 \mathrm{mM}$, giving $m=1.087$, is depicted as the red dashed line in Fig. 6. In fact, we previously found slight charge renormalization of uncorrelated, dilute colloids following Poisson-Boltzmann theory. ${ }^{16}$ Besides, for $c_{\mathrm{s}} \leq 8 \mathrm{mM}$ we also found significant charge renormalization as a function of colloid volume fraction, with $Z_{\text {eff }}$ values increasing with decreasing $\phi{ }^{16,35}$ If such charge renormalization would also occur at higher salt concentrations for real experimental colloids, albeit to a lesser degree, this would result in a tilting of the binodals with increasing repulsions, showing a larger increase at intermediate colloid volume fractions and a smaller increase at high colloid volume fractions. Unfortunately, theoretical descriptions of charge renormalization and regulation under strong screening conditions and high colloid volume fractions relevant to the extended GFVT remain to be developed.

For larger values of $m$ than those considered here we note that a coupling between electrostatics and polymer physics has been predicted to become relevant that is not included in our theoretical description. ${ }^{36}$ Indeed, within the validity of the extended GFVT, i.e. for $m$-values below 1.2 and $m^{1 / 3}<(1+\gamma),{ }^{8}$ these coupling effects are negligibly small close to the binodal. ${ }^{36}$ Care should be taken when applying GFVT to increasingly longranged repulsions.

\section{Phase diagrams with different $\gamma$}

Finally, we apply the same approach to colloids mixed with PEO of different sizes, giving $\gamma=0.25$ and 0.44 . We use different colloids for these measurements, though according to our SAXS characterization they have the same properties as the colloids listed in Table 1. The normalized results for $c_{\mathrm{s}}=50 \mathrm{mM}$ and 20 $\mathrm{mM}$ are shown in Fig. 7. Again we find quantitative agreement between the experimental onset of phase separation and the binodal predicted by the GFVT for repulsive colloids. This demonstrates the robustness of our model mixtures to study depletion interactions of charged colloids.

Since the tie lines for such small polymer/colloid size ratios are rather shallow, the gel line lies closer to the binodal. Although we have not studied this in detail, we also observe significant crystallization in mixtures just below the gel line. Therefore, it is not possible to measure sufficiently long dilution lines to apply the lever rule and to determine the composition of the coexisting phases of these mixtures. Although the directly measured binodal points at intermediate volume fractions correspond well with the theoretical predictions, deviations might occur at higher volume fractions, in line with our results for $\gamma=0.68$.

We have performed some explorative measurements of mixtures with smaller colloids and PEO with $R_{\mathrm{g}}=40 \mathrm{~nm}$ in 20 and $50 \mathrm{mM} \mathrm{NaCl}$ (results not shown). The composition of coexisting phases extracted with the lever rule seems to show the same tilt with decreasing salt concentration: a shift to relatively larger polymer concentrations at intermediate volume
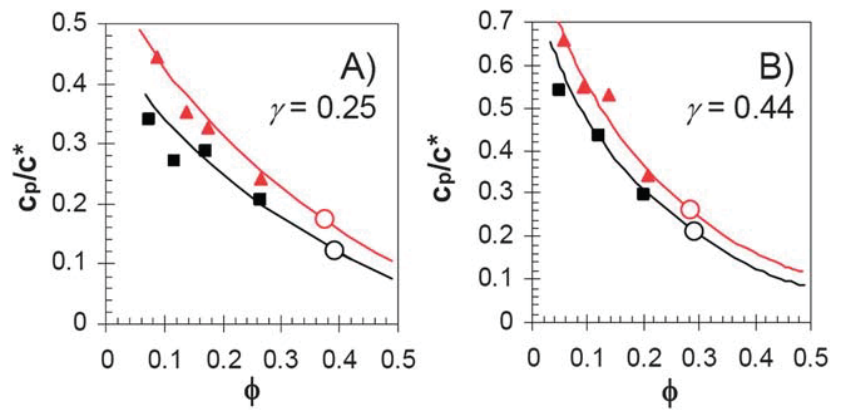

Fig. 7 Phase diagram with normalized axes for colloid-polymer mixtures with $c_{s}$ $=50 \mathrm{mM}$ (black squares and lines) and $20 \mathrm{mM}$ (red triangles and lines) and (A) $\gamma$ $=0.25$ and (B) $\gamma=0.44$. Filled symbols depict experimental binodal points, circles theoretical critical points, and solid lines GFVT results with $m=1.026$ (black) and $m=1.078$ (red) 
fractions and basically no effect at higher volume fractions. However, the SAXS measurements for these colloids could not be straightforwardly modelled with monodisperse charged colloids. Therefore, our results for $\gamma=0.68$ remain to be verified at higher values of $\gamma$ for equally well-defined colloid-polymer mixtures.

\section{Conclusions}

In conclusion, we have established binodals of aqueous mixtures of charged colloids and a neutral polymer as a function of salt concentration and polymer/colloid size ratio. For size ratios $\gamma=0.25,0.44$, and 0.68 , the experimental onset of phase separation can be quantitatively described by GFVT calculations for the binodal of charged spheres with excludedvolume polymers. For $\gamma=0.68$ and $m \approx 1.0$, we demonstrate that GFVT correctly describes the composition of coexisting phases, i.e. the tie lines, and the critical point. However, we find a qualitative deviation for the development of these tie lines with decreasing salt concentrations. We propose that this might be related to charge renormalization and regulation, which has barely been studied under high-colloid and high-salt conditions. These findings are highly relevant when one attempts to extrapolate the equilibrium phase diagram to explore nonequilibrium gelation at higher colloid and/or polymer concentrations in real systems.

\section{Acknowledgements}

We thank Peter Schurtenberger for valuable and encouraging discussions. Shirish Chodankar and Andreas Menzel are thanked for their help as local contacts for the SAXS measurements, which were financially supported by the European Commission under the Seventh Framework Program by means of the grant agreement for the Integrated Infrastructure Initiative no. 262348 European Soft Matter Infrastructure (ESMI). K.v.G. and A.S. gratefully acknowledge financial support from the Swiss National Science Foundation (SNF, 200021_119964), the University of Fribourg, and the Adolphe Merkle Foundation.

\section{Notes and references}

$1 \mathrm{H}$. N. W. Lekkerkerker and R. Tuinier, Colloids and the Depletion Interaction, Springer, Heidelberg, 2011.

2 S. Asakura and F. Oosawa, J. Chem. Phys., 1954, 22, 12551256.

3 A. Donald, Nat. Mater., 2004, 3, 579-581.

4 G. J. Fleer and R. Tuinier, Phys. Rev. E: Stat., Nonlinear, Soft Matter Phys., 2007, 76, 041802.

5 G. J. Fleer and R. Tuinier, Adv. Colloid Interface Sci., 2008, 143, 1-47.

6 H. N. W. Lekkerkerker, W. C. K. Poon, P. N. Pusey, A. Stroobants and P. B. Warren, Europhys. Lett., 1992, 20, 559-564.

7 C. Gogelein and R. Tuinier, Eur. Phys. J. E, 2008, 27, 171184.
8 A. Fortini, M. Dijkstra and R. Tuinier, J. Phys.: Condens. Matter, 2005, 17, 7783-7803.

9 J. A. Zhou, J. S. van Duijneveldt and B. Vincent, Langmuir, 2010, 26, 9397-9402.

10 A. P. Gast, W. B. Russel and C. K. Hall, J. Colloid Interface Sci., 1986, 109, 161-171.

11 O. Annunziata, N. Asherie, A. Lomakin, J. Pande, O. Ogun and G. B. Benedek, Proc. Natl. Acad. Sci. U. S. A., 2002, 99, 14165-14170.

12 M. Casselyn, J. Perez, A. Tardieu, P. Vachette, J. Witz and H. Delacroix, Acta Crystallogr., Sect. D: Biol. Crystallogr., 2001, 57, 1799-1812.

13 S. Finet and A. Tardieu, J. Cryst. Growth, 2001, 232, 40-49.

14 V. Y. Grinberg and V. B. Tolstoguzov, Food Hydrocolloids, 1997, 11, 145-158.

15 P. D. Patel and W. B. Russel, J. Colloid Interface Sci., 1989, 131, 192-200.

16 K. van Gruijthuijsen, M. Obiols-Rabasa, M. Heinen, G. Nägele and A. Stradner, Langmuir, DOI: 10.1021/la402104q.

17 J. C. Brown and P. N. Pusey, J. Phys. D: Appl. Phys., 1974, 7, L31-L35.

18 D. E. Koppel, J. Chem. Phys., 1972, 57, 4814-4820.

19 K. Devanand and J. C. Selser, Macromolecules, 1991, 24, 5943-5947.

20 K. W. Ebagninin, A. Benchabane and K. Bekkour, J. Colloid Interface Sci., 2009, 336, 360-367.

21 S. Kawaguchi, G. Imai, J. Suzuki, A. Miyahara and T. Kitano, Polymer, 1997, 38, 2885-2891.

22 S. Kinugasa, H. Nakahara, N. Fudagawa and Y. Koga, Macromolecules, 1994, 27, 6889-6892.

23 B. Vincent, P. F. Luckham and F. A. Waite, J. Colloid Interface Sci., 1980, 73, 508-521.

24 D. Vivares, L. Belloni, A. Tardieu and F. Bonnete, Eur. Phys. J. E, 2002, 9, 15-25.

25 P. N. Pusey and W. Van Megen, Nature, 1986, 320, 340-342.

26 L. J. Teece, M. A. Faers and P. Bartlett, Soft Matter, 2011, 7, 1341-1351.

27 C. P. Royall, W. C. K. Poon and E. R. Weeks, Soft Matter, 2013, 9, 17-27.

28 R. Tuinier, P. A. Smith, W. C. K. Poon, S. U. Egelhaaf, D. G. A. L. Aarts, H. N. W. Lekkerkerker and G. J. Fleer, Europhys. Lett., 2008, 82, 68002.

29 I. Bodnar, J. K. G. Dhont and H. N. W. Lekkerkerker, J. Phys. Chem., 1996, 100, 19614-19619.

30 W. C. K. Poon, A. D. Pirie and P. N. Pusey, Faraday Discuss., 1995, 65-76.

31 N. A. M. Verhaegh, D. Asnaghi, H. N. W. Lekkerkerker, M. Giglio and L. Cipelletti, Physica A, 1997, 242, 104-118.

32 E. Zaccarelli, P. J. Lu, F. Ciulla, D. A. Weitz and F. Sciortino, J. Phys.: Condens. Matter, 2008, 20, 494242.

33 W. C. K. Poon, J. Phys.: Condens. Matter, 2002, 14, R859R880.

34 E. J. W. Verwey and J. T. G. Overbeek, Theory of Stability of Lyophobic Colloids, Elsevier, Amsterdam, 1948.

35 N. J. Wagner, R. Krause, A. R. Rennie, B. D'Aguanno and J. Goodwin, J. Chem. Phys., 1991, 95, 494-508.

36 O. A. Croze and M. E. Cates, Langmuir, 2005, 21, 5627-5638. 